develop an abnormal glucose tolerance in pregnancy, but most often glucose tolerance returns to normal postpartum. This condition is called gestational diabetes mellitus (GDM).

Aims Comparative study between gestational and pregestational diabetes in relation to glycemic control as regarding fetal and neonatal outcome.

Methods This study was conducted in Kasralainy Maternity hospital in Egypt from september 2011 to march 2012 and it included 60 pregnant women complicated by DM attending outpatient clinic or inpatient. Patients were classified into two groups, Gestational Diabetes: 30 pregnant women complicated by DM which is diagnosed for the first time during pregnancy and Pregestational Diabetes: 30 pregnant women who have DM that has been diagnosed prior to pregnancy.

The two groups were compared according to fetal (macrosomia and intrauterine fetal death) and neonatal (respiratory distress syndrome and birth injuries) complications. All patients were 18 to 40 years old, singltone pregnancy, with time of termination after completed 37 weeks.

Results Fetal macrosomia occurred more with GDM, on the otherhand birth injuries and RDS occurred more with Pre-GDM. Macrosomia and RDS were commoner among poor glycemic control in pregnant diabetic females than birth injuries and IUFD.

Conclusions Glycemic control started as early as possible (the best being preconceptional) is important to decrease the incidence of birth injuries, macrosomia, fetal mortality, the need for NICU admission (RDS).

\section{PM.71 A CASE OF LATE ONSET POSTPARTUM ECLAMPSIA: AN EXTREME OF ECLAMPSIA?}

doi:10.1136/archdischild-2013-303966.152

S McGowan, K Connor, S McNeil. Altnagelvin Area Hospital, Western Health and Social Care Trust, UK, Londonderry, UK

Eclampsia is the onset of generalised seizures in the setting of pre-eclampsia. ${ }^{1}$ Traditionally eclampsia occurring more than 48 hours into the postnatal period was thought rare and termed; late onset postpartum eclampsia. ${ }^{2}$ Evidence shows a changing pattern of disease with eclampsia rates falling however a comparative increase in late postpartum seizures. ${ }^{3}{ }^{4}$ These may lack classical symptoms and signs and may not be identified before seizure onset. ${ }^{12}$

A 27 year old parous patient presented 7 days postnatally with generalised tonic-clonic seizures, severe hypertension and neurological irritability with no proteinuria. This was preceded acutely with severe headache. Previous history was uncomplicated by hypertensive disease and her partner was unchanged. There was no symptomatology of pre-eclampsia in the antenatal or immediately postnatal period. Antenatal history was uneventful showing generalised oedema only and there was concurrent and progressive fetal growth. Following routine discharge after caesarean section for failure to progress in labour this patient represented with a seizure from home. There was a normal CT of brain. This patient responded to magnesium sulphate and labetalol with no further seizures and a large diuresis. Ongoing blood pressure control proved to be difficult, requiring multispecialty input.

We conclude that the "classic" definitions of these diseases may present barriers for rapid diagnoses and treatment with late postpartum variants showing atypical symptomatology, signs and disease progression with a non-linear relationship between pre-eclampsia and eclampsia. We advocate increased vigilance and reporting in the late postpartum period.

\section{REFERENCES}

1. Munjuluri N, Lipman M, Valentine A, et al. Postpartum eclampsia of late onset. BMJ 2005;331:1070-1.
2. Dziewas $\mathrm{R}$, Stögbauer F, Freund $\mathrm{M}$, et al. Late onset postpartum eclampsia: a rare and difficult diagnosis. J Neurol 2002;249:1287-91.

3. Leitch CR, Cameron AD, Walker JJ. The changing pattern of eclampsia over a 60-year period. Br J Obstet Gynaecol 1997:104:917-22.

4. Chames MC, Livingston JC, Ivester TS, et al. Late postpartum eclampsia: a preventable disease? Am J Obstet Gynecol 2002;186:1174-7.

\section{PM.72 HOMOCYSTENEMIA AND PREGNANCY A DANGEROUS COMBINATION}

doi:10.1136/archdischild-2013-303966.153

S Ganta, V Ravimohan. Cumberland Infirmary Hospital, Carlisle, UK

Preeclampsia represents one of the most frequent complications of pregnancy. Damage of the endothelial layer lining the blood vessel wall is thought to play an important role in the pathophysiology of preeclampsia, accordingly, mild hyper homocysteinaemia has been reported to be more prevalent among preeclamptic women. Homocystine is a naturally occurring sulphur containing amino acid resulting from demethylation of methionine. The accumulation of homocysteine and its metabolites is caused by a disruption of any of the required enzymes or cofactors involved in the pathways of methionine metabolism. These abnormalities could arise from genetic predisposition and/or nutritional and environmental factors. The most common cause of homocysteinemia, encompassing $95 \%$ of the patients, is a deficiency in the cystathionine B-synthase (CBS) enzyme, defective methylcobalamin synthesis, or abnormality in methylene tetrahydrofolate reductase (MTHFR).Homocysteine is responsible for endothelial cell damage leading to proatherogenic effects, thromboembolic effects, hypoperfusion of placenta, and overproduction of free radicals. In pregnancy homocystenemia has been implicated with recurrent miscarriage,pre-eclamsia, placental abruption, IUGR, preterm delivery. We present a unique case of massive abruption and IUD. A 34 year old G4 P1 +2 with an uneventful antenatal period presented at 36 weeks with antepartum haemorrhage, massive abruption and intrauterine demise diagnosed on admission. Postpartum investigations of note were elevated urate at 394, red cell distance width of 19.2 , and Homocystine level of $17.7 \mathrm{mmol} / \mathrm{l}$ (mild Homocystinemia). Review of literature indicated plasma levels over $12 \mathrm{~mol} / \mathrm{L}$ should be treated aggressively with vitamin supplementation. We had therefore initiated therapy with B12, B6, folic acid to normalise homocysteine levels.

\section{PM.73 CASE REPORT: ABDOMINAL CUTANEOUS NERVE ENTRAPMENT SYNDROME IN THE PREGNANT PATIENT}

doi:10.1136/archdischild-2013-303966.154

C Dougan, P Campbell, D Sirivastava. Ulster Hospital Dundonald, Belfast, UK

Case Report A 25 yr para 1 (NVD) at $30+2$ weeks gestation presented with severe right sided abdominal pain. Vital signs were stable. Tenderness was elicited in the right lumbar region with voluntary guarding. WCC, CRP and urinalysis were normal. Fetal assessment ultrasound was normal. A Surgical opinion excluded acute surgical causes. Urgent MRI was normal. There was no improvement despite IV morphine. When the patient was reassessed, clinical examination revealed an area of tactile allodynia on the lateral edge of the rectus sheath from T8 to T12 with a positive Carnett's sign. A diagnosis of ACNES was made, and the pain resolved with administration of lignocaine patches. An exacerbation three weeks later required local infiltration of the cutaneous abdominal nerves. Caesarian delivery was performed at $34+5$ weeks to relieve abdominal distension and prevent risk to the fetus of opiate analgesia. The pain spontaneously resolved post-partum. 